Scientific Journal of October 6 University

ISSN (Print): 2314-8640

ISSN (Electronic): 2356-8119

Published by October 6 University @ All Rights Reserved

Available online at: http: sjou.journals.ekb.eg
Citation: Diaa El-Din et al., (2018). Development of an Optimal/Robust Control Strategy for Electrical Energy Cost Minimization in an AirConditioning Plant . Sci. J. of Oct. 6 Univ. 4 (2), 23-34.

Copyright: () 2018 Diaa El-Din et al. This is an open-access article distributed under the terms of the Creative Commons Attribution License, which permits unrestricted use, distribution, and reproduction in any medium, provided the original author and source are credited.

Original Article

\title{
Development of an Optimal/Robust Control Strategy for Electrical Energy Cost Minimization in an Air-Conditioning Plant
}

\author{
M.M. Diaa El-Din H. Farag, Farid A. Tolbah, Mohamed Osama Ezz El-Din
}

Ain Shams University, Faculty of Engineering, Cairo, Egypt.

\section{Received: 25-04-2018/ Revised: 10-05-2018 / Accepted: 20-06-2018}

\begin{abstract}
This paper focuses on modelling the thermal behaviour of buildings and designing an optimal control algorithm for their HVAC systems. The problem of developing a good model to capture the heat storage and heat transmission properties of building thermal elements such as rooms and walls is addressed by using the lumped capacitance method. The equations governing the system dynamics are derived using the thermal circuit approach, and by defining equivalent thermal masses, thermal resistors and thermal capacitors. In the control design part, we have introduced a control algorithm which is composed in Pontryagin's Minimum Principle controller. The optimal tracking problem is solved in Pontryagin's Minimum Principle controller where the interconnection of all walls are taken into consideration. The Pontryagin's Minimum Principle controller Ominimizes a quadratic cost function which has two quadratic terms. One takes into account the comfort level and the other represents the control effort, i.e. the energy consumed to operate the HVAC system. Simulation results for a single room example show energy savings using this control algorithm over a conventional PI controller.
\end{abstract}

Key Words: Pontryagin's Minimum Principle; Optimal Control;

\section{Introduction}

Systems of air-conditioning for countries founded in the tropics operate at a constant compressor speed as these countries experience a quite moderate diurnal temperature variation of the order of $5-10{ }^{0} \mathrm{C}$ throughout the year. The building's temperature is maintained constant using a simple on/off system to the air-handling unit. There was no proper control system is used in many cases to conserve energy. The selection of these systems for most application is mainly based on capital cost of the equipment and the use of a control system to conserve the electrical energy is not of prime importance.

In cases where there is need of an accurate temperature controller of an environment, for example, during manufacturing of electronic components, cooling and dehumidifying of air is accomplished through heating and cooling of air to the required conditions in the air handling unit.
Currently, there is a wide concern about the optimum use of energy in buildings. As the price of fuel has doubled in the last five years. Two topics were of general interests, they were Energy conservation and thermal comfort in buildings. Using well tuned controller for the air-handling unit (AHU) and variable-speed compressor (VSC) were suggested and investigated to maintain a thermal comfort of an environment room and to reduce the energy consumption from an airconditioning unit. Which also include the development of many different types of controller either for AHU or the compressor system. Among many control methods for heating and ventilation of air-conditioning application, the proportionalintegral-derivative (PID) algorithm is very common.

In the United States, homes and commercial buildings use $71 \%$ of the electricity according to the U.S. Energy Information Agency, and this 
number will rise to $75 \%$ by $2025[1]$. Homes account for $37 \%$ of all U.S. electricity consumption and $22 \%$ of all U.S. primary energy consumption (EIA 2005). This makes home energy reduction an important part of any plan to reduce U.S. contribution to global climate change [2].

\section{Mathematical Model}

Using the heat transfer equations fundamentals to derive the governing heat transfer equations for the temperature distribution in walls and room of a simple building. The heat transfer and storage equations compose a simple plant model representing a one room building Figure (1).

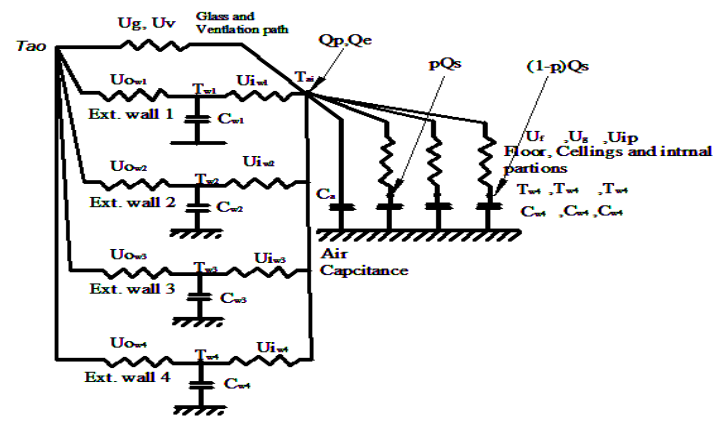

Figure 1: Lumped Capacitance Model of The Room

The model of room is based on a simple lumped capacity model. This model considers a building as a thermal network which is analogous to a electric circuit network. The nodes in the network are the components (room air, walls, ceiling etc.) which are considered as lumped capacitance. The temperature on each node is analogous to the voltage in a electric network. Heat flows among the nodes, just like the electric current. There is thermal resistance between components and thermal capacitance for each capacitance. Thus we get a linear thermal network and we can derive state-space equations through the energy balance of each node.

In our simple room building model, there are four walls which are identified by $\mathrm{w}_{1}, \mathrm{w}_{2}, \ldots, \mathrm{w}_{4}$. The area and the temperature of wall $i$ is called $A_{i}$ and $\mathrm{T}_{\mathrm{wi}}$ respectively. The temperature of the wall is assigned to its centre-line, separating the wall into two parts. The thermal capacity of a wall which is denoted by $\mathrm{C}_{\mathrm{wi}}$ may be defined as

$$
C_{w i}=\mathrm{m}_{w i} \mathrm{c}_{\mathrm{pw}}
$$

where the mass of wall $\mathrm{i}, \mathrm{m}_{\mathrm{i}}$ can be obtained from the following equation

$$
m_{\text {wi }}=\rho_{\mathrm{w}} \mathrm{V}_{\mathrm{wi}}
$$

Where $\rho_{\mathrm{w}}$ is the density of the walls and $\mathrm{V}_{\mathrm{wi}}$ is the volume of wall $i$ which is the area of the wall times its thickness.

Now we have one node for each the air inside the room, floor, ceiling, partitions and four nodes for the surrounding walls. These nodes should be linked to each other using the thermal resistances. Having the walls separated into two sides, we can define the thermal resistance for conduction for both sides of the wall. Therefore the thermal resistance for conduction for each side of the wall can be defined as

$$
R_{\text {cond, half }}=\frac{R_{w}}{2}
$$

where $R_{w}$ is the total thermal resistance of the wall, which can be expressed as

$$
R_{w}=\frac{L}{k A}
$$

Where $\mathrm{L}$ is the thickness of the wall, $\mathrm{k}$ is the thermal conductivity of the wall material, and A is the area of the wall.

the convective heat transfer coefficient (h) depends on the type of fluid, flow properties and temperature properties, it will have different values for the two sides of the walls depending on the factors mentioned above for each side[3]. For simplicity, we only consider two different convective heat transfer coefficients, one for the internal and one for the external sides of the peripheral walls. Notice that the internal walls have the same convective heat transfer coefficient on their both sides. We denote the internal convective heat transfer coefficient, by $h_{i}$ and the external convective heat transfer coefficient, by $h_{0}$. Accordingly the thermal resistance for convection on the internal and external sides of the peripheral walls denoted by $\left(\mathrm{R}_{\mathrm{i}}\right)$ and $\left(\mathrm{R}_{\mathrm{o}}\right)$, respectively, can be defined as follows

$$
\begin{aligned}
& R_{\mathrm{i}}=\frac{1}{h_{\mathrm{i}} A} \\
& R_{0}=\frac{1}{h_{0} A}
\end{aligned}
$$

Now we can derive the governing equation for the temperature evolution in walls, floor, ceiling, partitions, and air of the room, using the resistances and capacitors defined above. By performing nodal analysis we can get the following equation for the temperature of walls, floor, ceiling, partitions, and room air [4].

\subsection{Energy balance on external walls}

$$
\frac{\mathrm{dT}_{\mathrm{W}}}{\mathrm{dt}}=\frac{\mathrm{A}_{\mathrm{W}}}{\mathrm{C}_{\mathrm{W}}}\left[U_{w i}\left(\mathrm{~T}_{\mathrm{ai}}-\mathrm{T}_{\mathrm{w}}\right)+U_{w o}\left(\mathrm{~T}_{\mathrm{ao}}-\mathrm{T}_{\mathrm{w}}\right)\right]
$$

Where the subscripts refer to the number of the wall, for which the equation is written. The first term in he above equation accounts for the heat that is transferred from air in room to the wall. The second term represents the heat transfer from the outside to the wall. The term $\mathrm{U}_{\mathrm{wi}}$ and $\mathrm{U}_{\mathrm{wo}}$ represent the thermal transmittance of the wall 
inner and outer respectively, $\left(\mathrm{W} / \mathrm{m}^{2} \mathrm{~K}\right) . \mathrm{T}_{\mathrm{ao}},(\mathrm{K})$ outside air temperature, $\mathrm{T}_{\mathrm{ai}},(\mathrm{K})$ inside air temperature and $\mathrm{C}_{\mathrm{w}},(\mathrm{J} / \mathrm{K})$ thermal capacitance of the wall. A similar equation can be written for wall 2,3 and 4

We can write this equation for all of the walls of the building. So we have 4 equations governing the temperature evolution in the walls [5].

$$
\frac{2.2 \text { Floor }}{\mathrm{dt}}=\frac{\mathrm{A}_{\mathrm{f}}}{\mathrm{C}_{\mathrm{f}}}\left[\frac{\mathrm{pQ}_{s}}{\mathrm{~A}_{\mathrm{f}}}+U_{f}\left(\mathrm{~T}_{\mathrm{ai}}-T_{f}\right)\right]
$$

where $A_{f},\left(m^{2}\right)$ area of the floor, $C_{w},(J / K)$ thermal capacitance of the wall, $\mathrm{p}$ fraction of solar radiation entering floor, $\mathrm{Q}_{s}$, solar radiation through glazing, $U_{f}$

, $\left(\mathrm{W} / \mathrm{m}^{2} \mathrm{~K}\right)$ thermal transmittance of the floor and $\mathrm{T}_{\mathrm{f}},\left({ }^{\circ} \mathrm{C}\right)$ temperature of floor.

2.3 Ceiling

$$
\frac{\mathrm{dT}_{\mathrm{c}}}{\mathrm{dt}}=\frac{\mathrm{A}_{\mathrm{c}}}{\mathrm{C}_{\mathrm{c}}}\left[\mathrm{U}_{\mathrm{c}}\left(\mathrm{T}_{\mathrm{ai}}-\mathrm{T}_{\mathrm{c}}\right)\right]
$$

where $A_{c},\left(m^{2}\right)$ area of the ceiling, $C_{c},(J / K)$ thermal

$$
U_{c}
$$

capacitance of the ceiling, , $\left(\mathrm{W} / \mathrm{m}^{2} \mathrm{~K}\right)$ thermal transmittance of the ceiling and $\mathrm{T}_{\mathrm{c}},\left({ }^{\circ} \mathrm{C}\right)$ temperature of ceiling.

\subsection{Partitions}

$$
\frac{\mathrm{dT}_{\mathrm{ip}}}{\mathrm{dt}}=\frac{\mathrm{A}_{\mathrm{ip}}}{\mathrm{C}_{\mathrm{ip}}}\left[\frac{(1-\mathrm{p}) \mathrm{Q}_{\mathrm{s}}}{\mathrm{A}_{\mathrm{ip}}}+\mathrm{U}_{\mathrm{ip}}\left(\mathrm{T}_{\mathrm{ai}}-\mathrm{T}_{\mathrm{ip}}\right)\right]
$$

where $A_{i p},\left(m^{2}\right)$ area of the internal partition, $C_{i p}$, $(\mathrm{J} / \mathrm{K})$ thermal capacitance of internal partition, $\mathrm{p}$ fraction of solar radiation entering floor, $\mathrm{Q}_{\mathrm{s}}$, solar

$$
U_{i p}
$$

radiation through glazing, , $\left(\mathrm{W} / \mathrm{m}^{2} \mathrm{~K}\right)$ thermal transmittance of internal partition and $\mathrm{T}_{\mathrm{ip}},\left({ }^{\circ} \mathrm{C}\right)$ temperature of internal partition.

2.5 Air

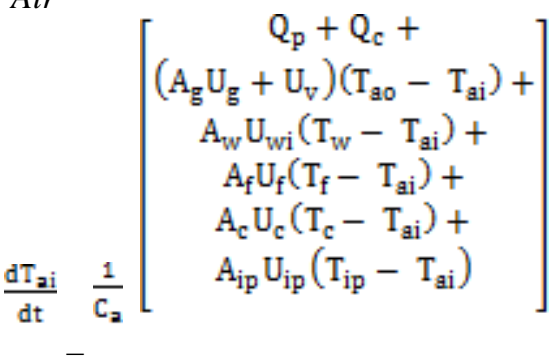

where $C_{a},(J / K)$ thermal capacitance of air,

$$
\mathrm{Q}_{\mathrm{C}}
$$

heat supplied by the plant, , (W) is the heat generation inside the room which can be from electrical devices such as computers, or from humans, lighting and etc.

If we write the heat transfer equation for every wall and room in the building and represent the equations in a state space form we get the following form of equation.

$$
\mathrm{X}=\mathrm{AX}+\mathrm{BU}
$$

$$
\mathrm{Y}=\mathrm{CX}
$$

Where $\mathbf{X}^{\cdot}$ is a vector of derivatives of temperatures, $\mathbf{A}, \mathbf{B}$ are matrices of coefficients, $\mathbf{X}$ is a vector of states and $\mathbf{U}$ is the input vector. We select the temperatures of walls $\left(\mathbf{T}_{\mathbf{w n}}\right)$, ceiling $\left(\mathbf{T}_{\mathbf{c}}\right)$, floor $\left(\mathbf{T}_{\mathbf{f}}\right)$, room air $\left(\mathbf{T}_{\mathbf{a i}}\right)$, etc. as state variables in $\mathbf{X}(\mathbf{t})$. And the input variables in $\mathbf{U}(\mathbf{t})$ include heat supplied by the plant $\left(\mathbf{Q}_{\mathbf{p}}\right)$, internal "casual" heat gains $\left(\mathbf{Q}_{\mathrm{c}}\right)$, solar radiation through windows $\left(\mathbf{Q}_{\mathrm{s}}\right)$, and outside air temperature $\left(\mathbf{T}_{\mathbf{a o}}\right)$. 


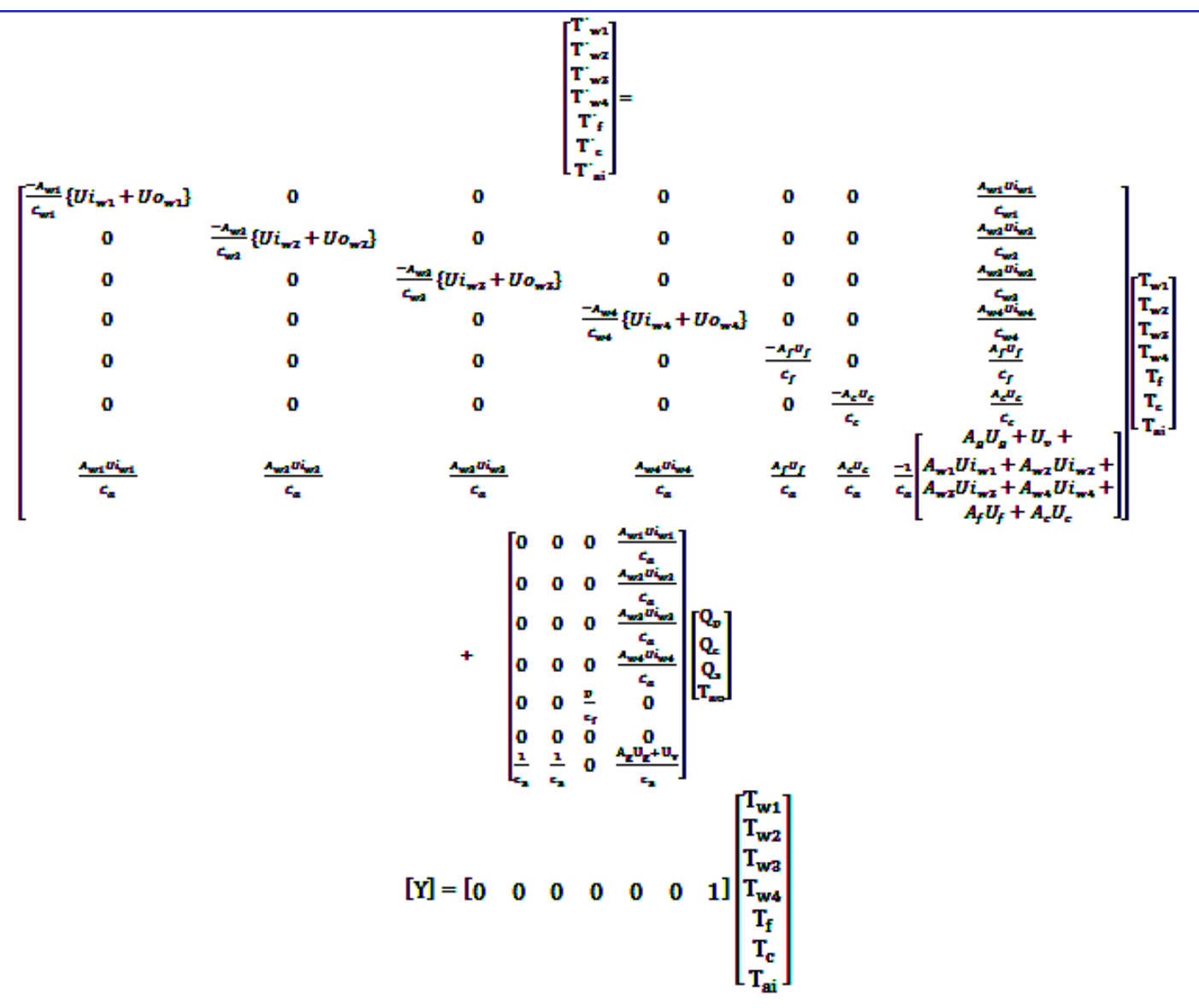

The construction materials and properties are selected according to ASHRAE handbook .The U-values (which are the reciprocal of the thermal resistance) and thermal capacitance are calculated, as shown in Table1.

\begin{tabular}{|c|c|c|c|c|c|c|c|c|c|}
\hline & $\begin{array}{c}\mathrm{A} \\
(\mathrm{ft2})\end{array}$ & $L(f t)$ & Rho (Tb/ft3) & $\mathrm{M}(\mathrm{Ib})$ & $\mathrm{cp}\left(\mathrm{BTU} / \mathrm{Tb}{ }^{\circ} \mathrm{F}\right)$ & Uo & W & $\mathbf{U}$ & $\mathrm{C}\left(\mathrm{BTU} /{ }^{\circ} \mathrm{F}\right)$ \\
\hline Front wall & 179 & 033 & 120 & 7088 & 0.19 & $\underline{2.60}$ & $\underline{0.056}$ & 0.06 & 1346.80 \\
\hline Back wall & 200 & 0.33 & 120 & 7920 & 0.19 & $\underline{2.60}$ & $\underline{0.056}$ & 0.06 & 1504.8 \\
\hline Left wall & 150 & 033 & 120 & 5940 & 0.19 & $\underline{2.60}$ & $\underline{0.056}$ & 0.06 & 1128.6 \\
\hline Right wall & 150 & 033 & 120 & 5940 & 0.19 & $\underline{2.60}$ & $\underline{0.056}$ & 0.06 & 1128.6 \\
\hline Floor & 300 & 0.5 & 37 & 5550 & 0.6 & 0.24 & 0.24 & 0.14 & 3330 \\
\hline Ceiling & 300 & & & 4500 & 0.6 & 0.19 & 0.17 & 0.096 & 2700 \\
\hline
\end{tabular}


As shown in previous modelling results, we have a seven-order system and it is difficult to deal with it in later control strategy steps. So, the next step is to reduce our system model order by using some reduction techniques which will be illustrated in the next section model order reduction.

\section{Model Order Reduction}

As shown in previous section, it is deduced that our seven-order system is very complex to be simulated numerically as the complexity of the control law often depends on the order of the system.

For control design purposes, we can approximate the model with another model of reduced order that preserves the original transfer function as much as possible.This can be done by eliminating uncontrollable and unobservable modes that do not affect the transfer function by using two subsequent stages, "Balanced Transformation" is used to transform our unbalanced system to balanced system and then we make "Model Reduction" to reduce our obtained balanced system to real reduced system that maintain the same transfer function as the original system behaviour.

Actually, we have two options of techniques for reducing our system order and the preferred technique is chosen according to its resulting deviation from the original behaviour. Techniques that will be tested are "DELETE Technique" and "MATCH DC Technique"

\subsection{Delete Technique}

This technique reduces the order of the model by eliminating the states associated with small oi's using the following MATLAB function.

rsys $=\operatorname{modred}\left(\operatorname{sysb}, e l i m,{ }^{\prime}{ }^{\prime} l^{\prime}\right)$

$$
\begin{aligned}
& \mathrm{X}^{\prime}=\mathrm{A} \mathrm{X}+\mathrm{B} \mathrm{U} \\
& {\left[\begin{array}{l}
\mathrm{x}_{1}^{s} \\
\mathrm{x}_{2}^{s}
\end{array}\right]=\left[\begin{array}{cc}
-0.0241 & 0.1212 \\
0.1212 & -1.1432
\end{array}\right]\left[\begin{array}{l}
\mathrm{x}_{1} \\
\mathrm{x}_{2}
\end{array}\right]}
\end{aligned}
$$
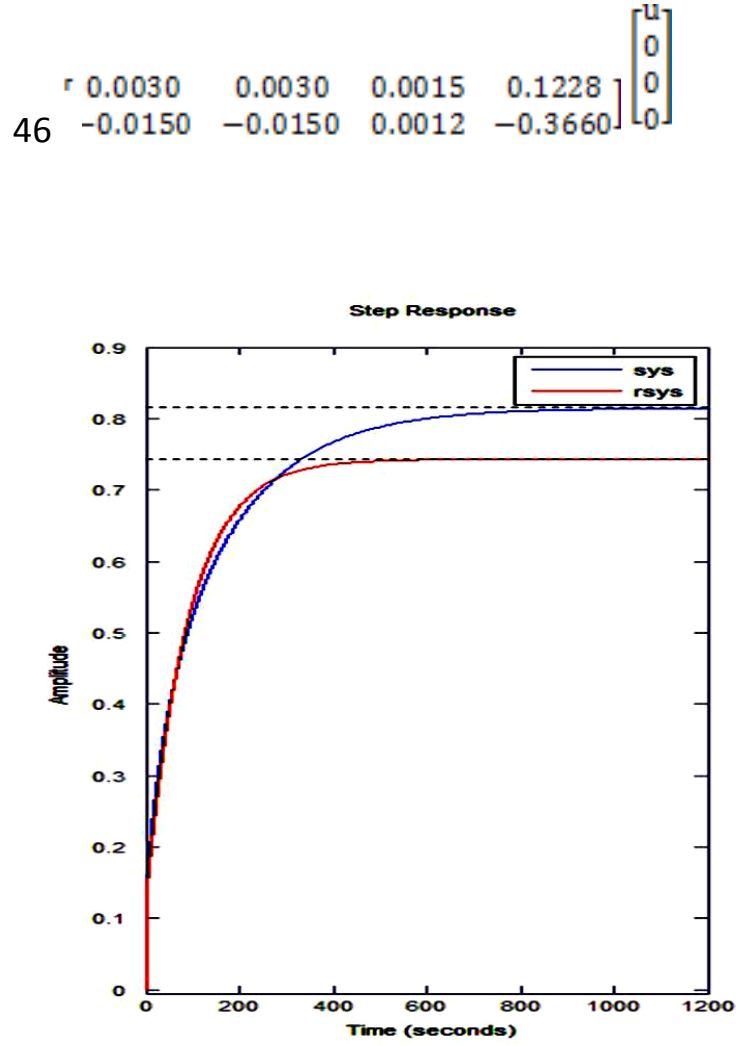

Figure 2: The step response is showing the difference between the original system (sys) and reduction system(rsys)

We observed in figure 2 that the behaviour of the reduced system differs from the original system behaviour with a considerable error. So, we will go ahead with another technique "MatchDC Technique".

\subsection{MatchDC Technique}

The matched DC-gain method preserves the DC gain of the original full-order model using the following simple function
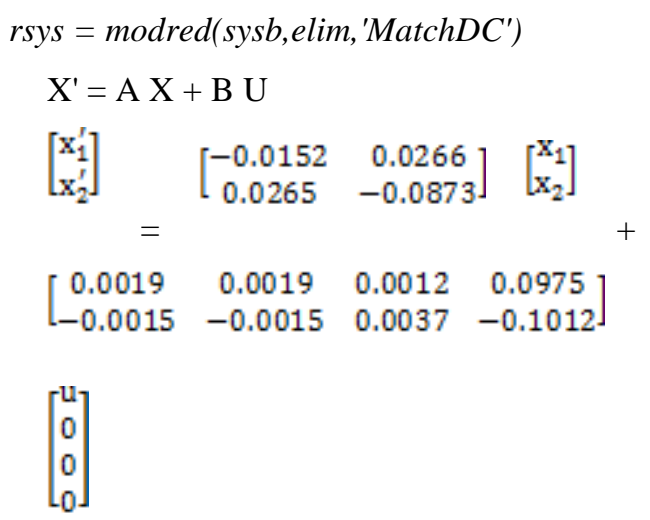


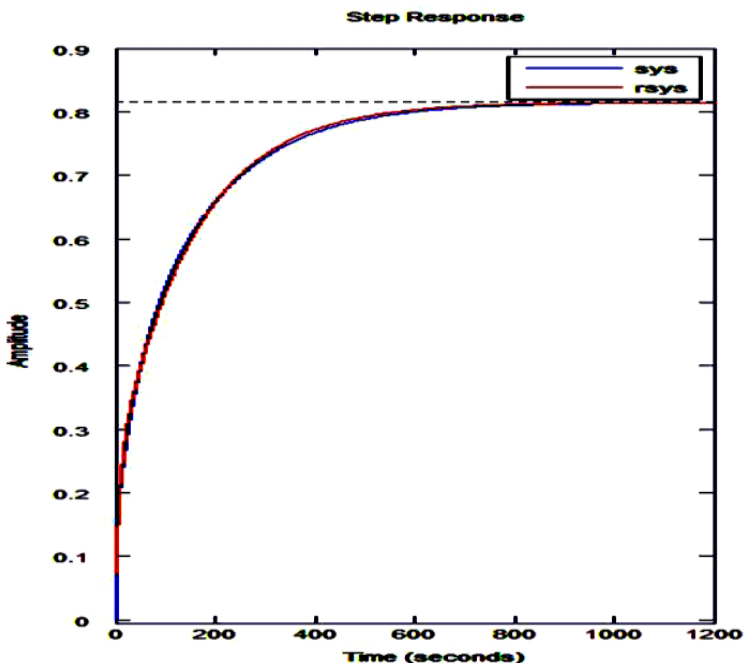

Figure 3: The step response is showing there are no difference between the original system (sys) and reduction system(rsys)

Using the "MatchDC" technique, the behaviour deviation error is approximately negligible and cannot be considered as shown in figure 3 .

So we will accept "MatchDC" result to continue with it in next section Dynamic Optimization, using Pontryagin's Minimum Principle which relies on second order equations obtained from "MatchDC" technqiue.

\section{Dynamic Optimization}

Dynamic Optimization interested in optimization of plants under dynamic conditions, i.e., the system variables are variant with respect to time and consequently the time is included in system representation. Then the plant is represented by differential equations. Techniques involved are calculus of variations, dynamic programming, search techniques, and Pontryagin technique principle.

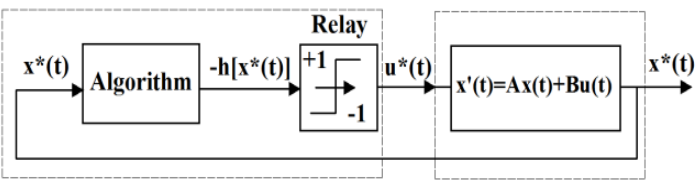

Closed-Loop Time-Optimal Controller

Plant

Figure 4: Closed-Loop Structure for Time-Optimal Control System

\subsection{Optimal Control}

The main target of optimal control is determining control signals that will drive plant to accomplish some constraints and at the same time extremize (i.e. maximize or minimize) cost function. We are aiming to getting the optimal control signal $\mathrm{u}^{*}(\mathrm{t}) \mathrm{H}^{*}$ denotes optimal condition) to drive the plant $\mathrm{P}$ from initial state to final state with some constraints on controls and states and at the same time extremizing the cost function $\mathrm{J}$.

The description of optimal control problem requires:

1. a mathematical model that describes the process to be controlled (i.e. state variables matrix),

2. determining the cost function, and

3. a specification of boundary conditions and the constraints on the states and/or controls.

\subsection{Time-Optimal Control of LTI System}

In this section, we address the problem of minimizing the time taken for the system to go from an initial state to the desired final state of a linear, time-invariant (LTI) system. The desired final state can be conveniently taken as the origin of the state space; in this way we will be dealing with time-optimal regulator system.

\subsection{Problem Formulation and Statement}

Let us now present a typical time-optimal control (TOC) system. Consider a linear, time-invariant dynamical system

$$
x^{\cdot}(t)=A x(t)+B u(t)
$$

where, $x(t)$ is $n$th state vector; $u(t)$ is $r$ th control vector, and the matrices $\mathrm{A}$ and $\mathrm{B}$ are constant matrices of nxn and nxr dimensions, respectively. We are also given that

1. the system (18) is completely controllable, that is, the matrix 
$G=\left[\begin{array}{lllllllll}B & \vdots & A B & \vdots & A^{2} B & \vdots & \cdots & \vdots & A^{n-1} B\end{array}\right]$

is of rank $\mathrm{n}$ or the matrix $\mathrm{G}$ is non-singular, and

2. the magnitude of the control $u(t)$ is constrained as

$$
U_{-} \leq u(t) \leq U_{+} \quad|u(t)| \leq U
$$

Here, U+ and U- are the upper and lower bounds of U. But, the constraint relation (20) can also be written more conveniently (by absorbing the magnitude $U$ into the matrix $B$ ) as

$$
\begin{aligned}
& -1 \leq u(t) \leq+1 \\
& |u(t)| \leq 1
\end{aligned}
$$

3. the initial state is $x\left(t_{0}\right)$ and the final (target) state is 0

The problem statement is: Find the (optimal) control $\mathrm{u}^{*}(\mathrm{t})$ which satisfies the constraint (21) and drives the system (18) from the initial state $\mathrm{x}\left(\mathrm{t}_{\mathrm{o}}\right)$ to the origin 0 in minimum time.

\subsection{Solution of the TOC System}

We develop the solution to this time-optimal control (TOC) system stated previously under the following steps. First let us list all the steps here and then discuss the same in detail.

- $\quad$ Step 1: Cost Function

- Step 2: Hamiltonian

- $\quad$ Step 3: Minimization of Hamiltonian

- $\quad$ Step 4: Costate Solutions

- $\quad$ Step 5: Time-Optimal Control Sequences

- $\quad$ Step 6: State Trajectories

- $\quad$ Step 7: Switch Curve

- $\quad$ Step 8: Phase Plane Regions

- $\quad$ Step 9: Control Law

- $\quad$ Step 10: Minimum Time

\section{Results}

\subsection{Introduction}

In the present study we demonstrate the way to obtain the closed loop structure for time-optimal control system of a second order (double integral) AHU system.

\subsection{TOC of a Double Integral System (AHU)}

Here we examine the time-optimal control (TOC) of AHU system which demonstrates a well-known procedure starting from problem formulation and statement then define our problem solution steps passing through implementation of control law.

\subsubsection{Problem Formulation and Statement}

\subsubsection{Problem Formulation}

AHU system is described by second order equations as illustrated earlier, recalling we reduced our seven order system to a simple second order system using MATCHDC technique. $\mathrm{X}^{\prime}=\mathrm{A} \mathrm{X}+\mathrm{B} \mathrm{U}$

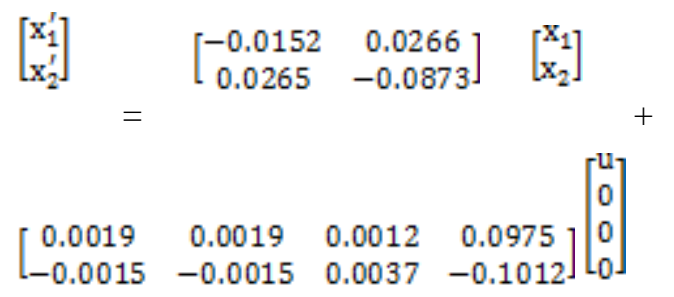

$$
\frac{d x_{1}(t)}{d t}=-0.0152 x_{1}(t)+0.0266 x_{2}(t)+0.0019 u(t)
$$

$$
\begin{aligned}
\frac{d x_{2}(t)}{d t} & =0.0265 x_{1}(t)-0.0873 x_{2}(t)-0.0015 u(t) \\
\mathrm{x}_{1}, \mathrm{x}_{2} &
\end{aligned}
$$

where, derivatives of walls temperatures;

$$
\mathrm{x}_{1}(\mathrm{t}), \mathrm{x}_{2}(\mathrm{t}) \quad u(t) \text { walls temperatures and }{ }^{\mathrm{is} \text { input }}
$$

signal represented by heat supplied by the plant $\left(Q_{p}\right)$.

$$
u(t)
$$

The control input to the system is constrained as

$$
|u(t)| \leq 1 \forall t \in\left[t_{0}, t_{1}\right]
$$




\subsubsection{Problem Statement}

Given the double integral system and the constraint on the control find the admissible control that forces the system from any initial state $\left[\mathrm{x}_{1}(0), \mathrm{x}_{2}(0) \quad\right]$ to the origin in minimum time.

Let us assume that we are dealing with normal system and no singular controls are allowed. Now, we attempt to solve the system following the procedure described earlier.

\subsubsection{Problem Solution}

Our problem solution consists of the list of the following steps with the details following.

- Step 1: Cost Function : For minimum-time system, the cost function is easily seen to be

$$
l=\int_{t_{0}}^{t_{f}} 1 d t=t_{f}-t_{0}
$$

where, $t_{0}$ is fixed and $t_{f}$ is free.

- Step 2: Hamiltonian: From the system (22), (23) and the cost function (25), form the Hamiltonian

$$
x(t), u(t), \lambda(t)
$$

$$
\begin{aligned}
& \mathrm{H}(\mathrm{l}) \quad=\quad 1+ \\
& \lambda_{1}(t)\left[-0.0152 x_{1}(t)+0.0266 x_{2}(t)\right] \\
& \lambda_{2}(t) \quad x_{1}(t) \quad x_{2}(t) \\
& {\left[\begin{array}{lll}
0.0265 & -0.0873 & ]
\end{array}\right]+0.0019} \\
& \lambda_{1}(t) u(t) \quad \lambda_{2}(t) u(t) \\
& -0.0015
\end{aligned}
$$

- Step 3: Minimization of Hamiltonian: According to the Pontryagin Principle, we need to minimize the Hamiltonian as

$$
\mathrm{H}\left(x^{*}(t), \lambda^{*}(t), u^{*}(t)\right)
$$

$$
\leq
$$

$$
\begin{gathered}
H\left(x^{*}(t), \lambda^{*}(t), u(t)\right) \\
=\quad \min _{|u| \leq 1} H\left(x^{*}(t), \lambda^{*}(t), u(t)\right)
\end{gathered}
$$

Using the Hamiltonian (26) in the condition (27), we have

$$
\begin{gathered}
1+\lambda_{1}^{*}(t)\left[-0.0152 x_{1}^{*}(t)+0.0266 x_{2}^{*}(t)\right]+\lambda_{2}^{*}(t)\left[0.0265 x_{1}^{*}(t)-0.0\right. \\
-0.0015 \lambda_{2}^{*}(t) u^{*}(t) \\
\leq \\
1+\lambda_{1}^{*}(t)\left[-0.0152 x_{1}^{*}(t)+0.0266 x_{2}^{*}(t)\right]+\lambda_{2}^{*}(t)\left[0.0265 x_{1}^{*}(\right. \\
-0.0015 \lambda_{2}^{*}(t) u(t)
\end{gathered}
$$

which leads to

$$
\begin{aligned}
& {\left[0.0019 \lambda_{1}^{*}(t)-0.0015 \lambda_{2}^{*}(t)\right] u^{*}(t)} \\
& \leq \\
& \left.0.0019 \lambda_{1}^{*}(t)-0.0015 \lambda_{2}^{*}(t)\right] u(t)
\end{aligned}
$$

Using the result of the previous section, we have the optimal control given in terms of the signum function as

$$
\begin{aligned}
& \mathrm{u}^{*}(\mathrm{t})=-\mathrm{SGN}\{\mathrm{q}(\mathrm{t})\} \\
& \mathrm{u}^{*}(\mathrm{t})=-\mathrm{SGN}\left\{0.0019 \lambda_{1}^{*}(t)-0.0015 \lambda_{2}^{*}(\mathrm{(t)})\right\}
\end{aligned}
$$

Now to know the nature of the optimal control, we need to solve for the costates function $\lambda_{1}^{*}(t)$ and $\lambda_{2}^{*}(t)$

- Step 4: Costate Solutions: The costate equations along with the Hamiltonian (26) are

$$
\begin{gathered}
\lambda_{1}^{*}(t)=-\frac{\partial \mathrm{H}}{\partial x_{1}^{*}} \\
=-\left[-0.0152 \lambda_{1}^{*}(t)+0.0265 \lambda_{2}^{*}(t)\right] \\
\lambda_{2}^{*}(t)=-\frac{\partial \mathrm{H}}{\partial x_{2}^{*}}
\end{gathered}
$$




$$
=-\left[0.0266 \lambda_{1}^{*}(t)-0.0873 \lambda_{2}^{*}(t)\right]
$$

Solving the previous equations, we get the costates as

$$
\begin{aligned}
& \lambda_{1}^{*}(t)=\lambda_{1}^{*}(0) e^{0.0152 t}+\lambda_{2}^{*}(0) e^{-0.0265 t} \\
& \lambda_{2}^{*}(t)=\lambda_{1}^{*}(0) e^{-0.0266 t}+\lambda_{2}^{*}(0) e^{0.0973 t}
\end{aligned}
$$

singularity: singular interval cannot exist because the function "q" can only be zero if the initial

$$
\begin{array}{ll}
\text { costates are equal zero i.e. } & \lambda_{1}^{*}(0) \\
\lambda_{2}^{*}(0) & =0 \text {, then } \\
\lambda_{1}^{*}(t) & \lambda_{2}^{*}(t)
\end{array}
$$
the costates leads to equal zero i.e. = $=0$ and Hamiltonian condition will be equal one, however in NTOC, Hamiltonian condition must equal zero; pontryagin and his co-workers derived that condition [7].

by substituting (33),(34) in the function "q" we get

$$
\begin{aligned}
& q(t)=\left[0.0019 \lambda_{1}^{*}(t)-0.0015 \lambda_{2}^{*}(t)\right] \\
& = \\
& \lambda_{1}^{*}(0)\left[0.0019 e^{0.0152 t}-0.0015 e^{-0.0266 t}\right]+ \\
& \lambda_{2}^{*}(0)\left[0.0019 e^{-0.0265 t}-0.0015 e^{0.0973 t}\right. \\
& ]
\end{aligned}
$$

- Step 5: Time-Optimal Control Sequences:

From the solutions of the costates (33) and (34),

$$
\lambda_{1}^{*}(t) \quad \lambda_{2}^{*}(t)
$$

we see that and are parabolic lines, and that there are four possible (assuming initial

$$
\lambda_{1}^{*}(0) \quad \lambda_{2}^{*}(0)
$$

conditions and to be nonzero) solutions as shown in Figure 5. Also shown are the four possible optimal control sequences

$$
\{+1\},\{-1\},\{+1,-1\},\{-1,+1\}
$$

that satisfy the optimal control relation (30). Let us reiterate that the admissible optimal control sequences are the ones given by (36). That is, a control sequence like $\{+1,-1,+1\}$ is not an optimal control sequence. Also, the control sequence $\{+$ $1,-1,+1\}$ requires two switching which is in violation of the earlier result that a second (nth) order system will have at most $1(\mathrm{n}-1)$ switching. From Figure 5, we see that the time-optimal control for the second order (double integral) system is a piecewise constant and can switch at most once. In order to arrive at closed-loop realization of the optimal control, we need to find the phase ( ) plane (state) trajectories.

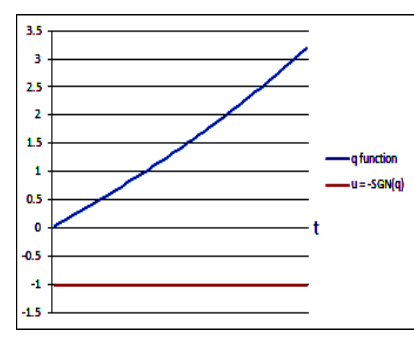

$\lambda_{1} \quad \lambda_{2}$

(a) (0) $>0$;

$(0)<0$ (b)

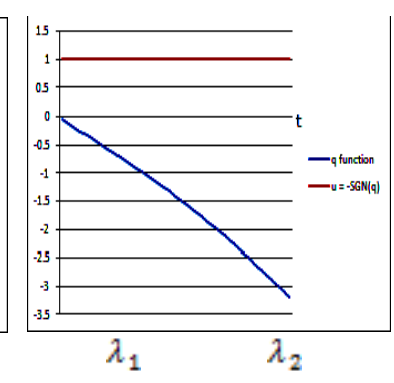

(0) $<0$;

(0) >

0

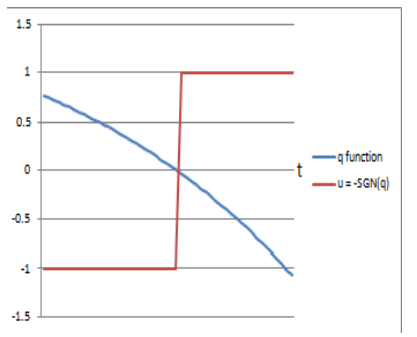

$\lambda_{1} \quad \lambda_{2}$
(0) $<0$

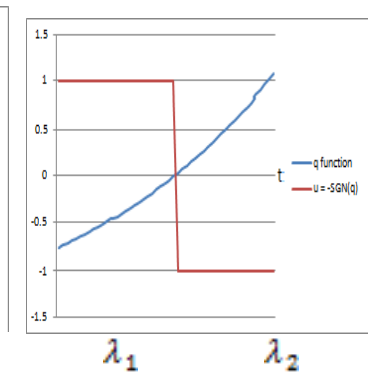

(d) (0) $>0$;

(0) >

0

Figure 5: Possible Costates and the Corresponding Controls

- Step 6: State Trajectories: Solving the state equations (23), we have

$$
x_{1}^{*}(\mathrm{t})=\frac{1}{1.107}\left[c_{1} \mathrm{e}^{-0.0065 \mathrm{t}}-0.328 c_{2} \mathrm{e}^{-0.096 \mathrm{t}}+0.223 \mathrm{U}\right]
$$

$x_{2}^{*}(t)=\frac{1}{1.107}\left[0.328 c_{1} \mathrm{e}^{-0.0065 t}+c_{2} \mathrm{e}^{-0.096 \mathrm{t}}+0.0487 \mathrm{U}\right]$

\section{$u^{*}$}

where, $\mathrm{U}=(\mathrm{t})= \pm 1$. For phase plane plots, we need to eliminate $t$ from solutions (37) for the 
states. Thus, (for simplicity, we omit $*$ since we are now dealing with all optimal functions only.

$$
\mathrm{t}=-533 \ln \left(\bar{F}_{\mathrm{x}_{1}}(t)+0.201\right)
$$

$$
\left(x_{1}, x_{2}\right)
$$

transfer any initial state

to the origin $(0,0)$.

1. The $\gamma_{+}$curve is the locus of all (initial) points $\left(x_{1}, x_{2}\right)$

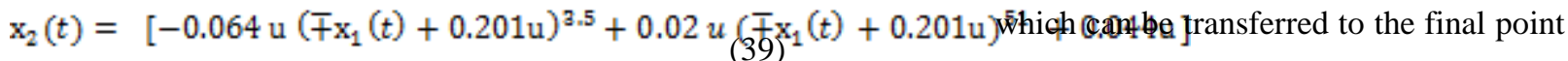
$(0,0)$ by the control $u=+1$. That is

where, we used $U= \pm 1$. If

$\mathrm{u}=\mathbb{U}=+1_{s} \quad\left\{\begin{array}{c}\mathrm{t}=-533 \ln \left(-\mathrm{x}_{1}(\mathrm{t})+0.201\right) \\ \mathrm{x}_{2}(\mathrm{t})=\left[-0.064\left(-\mathrm{x}_{1}(\mathrm{t})+0.201\right)^{\mathrm{a} .5}(40 .) 044\right]\end{array}\right.$

$$
\begin{aligned}
& \gamma_{+}=\left[\left(x_{1}, x_{2}\right):\right. \\
& \mathrm{x}_{2}=\left[-0.064\left(-\mathrm{x}_{1}+0.201\right)^{2.5}+0.044\right],
\end{aligned}
$$

And if

$\mathrm{u}=\mathrm{U}=-1_{s} \quad\left\{\begin{array}{c}\mathrm{t}=-533 \ln \left(\mathrm{x}_{1}(\mathrm{t})+0.201\right) \\ \mathrm{x}_{2}(\mathrm{t})=\left[0.064\left(\mathrm{x}_{1}(\mathrm{t})+0.201\right)^{\mathrm{a} .5}-0.044\right] \text {. The } \gamma \text {. curve is the locus of all (initial) points }\end{array}\right.$ Now, we can easily see that the relations (40) and $\left(x_{1}, x_{2}\right)$ which can be transferred to the final point (41) represent a family of parabolas in $($, ) plane (or phase plane) as shown in Figure 6. The arrow indicates the direction of motion for increasing (positive) time. Our aim is to drive the

$$
x_{1}(0) x_{2}(0)
$$

system from any initial state (, ) to origin $(0,0)$ in minimum time.

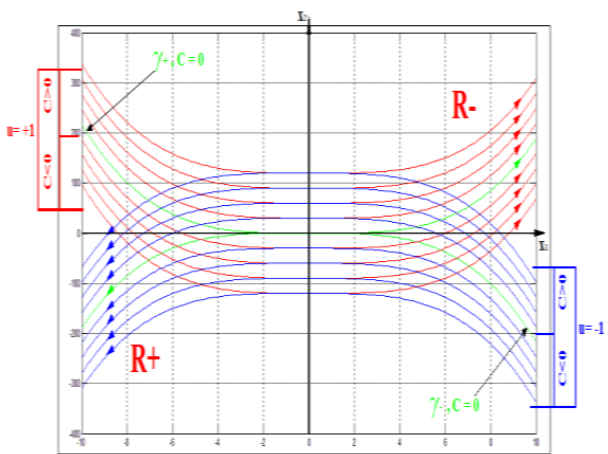

Figure 6: Phase Plane Trajectories for $\mathrm{u}=+1$ (red lines) and $\mathrm{u}=-1$ (blue lines)

Now we can restate our problem as to find the time-optimal control sequence to drive the system

$$
x_{1} x_{2}
$$

from any initial state $(, \quad)$ to the origin $(0,0)$ in minimum time.

- Step 7: Switch Curve: From Figure 6, we see that there are two curves labeled $\gamma_{\text {- }}$ and $\gamma_{+}$which
$(0,0)$ by the control $u=-1$. That is

$$
\gamma_{-}=\left[\left(x_{1}, x_{2}\right):\right.
$$

$\left.x_{2}=\left[0.064\left(x_{1}+0.201\right)^{a .5}-0.044\right], \quad x_{1} \geq 0\right]$

3. The complete switch curve, i.e., the $\gamma$ curve, is defined as the union (either or ) of the partial switch curves $\gamma_{+}$and $\gamma_{-}$. That is

$$
\begin{aligned}
& y=\left[\left(x_{1}, x_{2}\right):\right. \\
& \left.\mathrm{x}_{2}=\left[0.064\left(\mathrm{x}_{1}+\operatorname{sgn}\left(\mathrm{x}_{1}\right) 0.201\right)^{2.5}-\operatorname{sgn}\left(\mathrm{x}_{4}\right) 0.044\right]\right] \\
& =\gamma_{+} \cup \gamma_{-}
\end{aligned}
$$

where, $\mathrm{U}$ means the union operation.

The switch curve $\gamma$ is shown in Figure 7. 


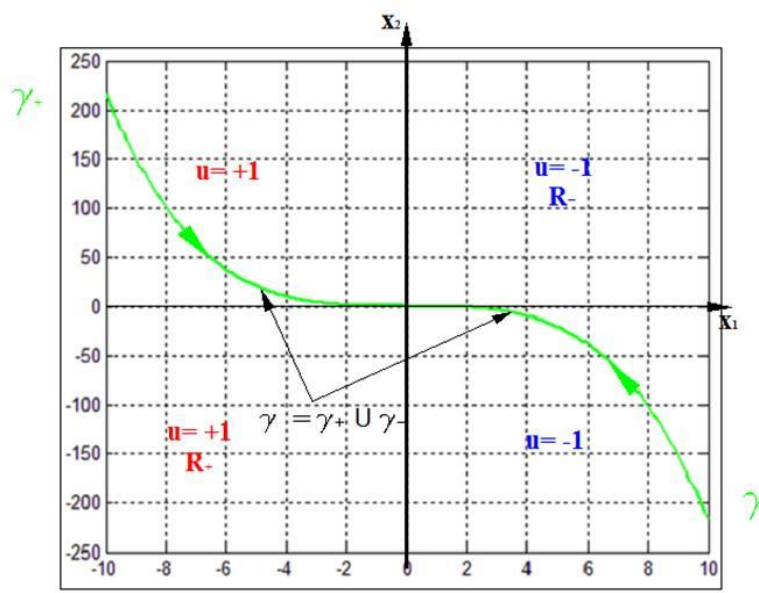

Figure 7: Switch Curve for Double Integral TimeOptimal Control System

- Step 8: Phase Plane Regions: Let us now define the regions in which we need to apply the control $u=+1$ or $u=-1$.

1. Let $R_{+}$be the region of the points such that

$R_{+}=\left[\left(x_{1}, x_{2}\right)\right.$ :

$x_{2}<\left[0.064\left(x_{1}+\operatorname{sgn}\left(x_{1}\right) 0.201\right)^{a .5}-\operatorname{sgn}\left(x_{1}\right) 0.044\right]$

That is R consists of the region of the points to the left of the switch curve $\gamma$.

2. Let R be the region of the points such that

$R_{-}=\left[\left(x_{1}, x_{2}\right)\right.$ :

$x_{2}>\left[0.064\left(x_{1}+\operatorname{sgn}\left(x_{1}\right) 0.201\right)^{3.5}-\operatorname{sgn}\left(4 x_{1}\right)\right.$

That is $\mathrm{R}$ - consists of the region of the points to the right of the switch curve $\gamma$.

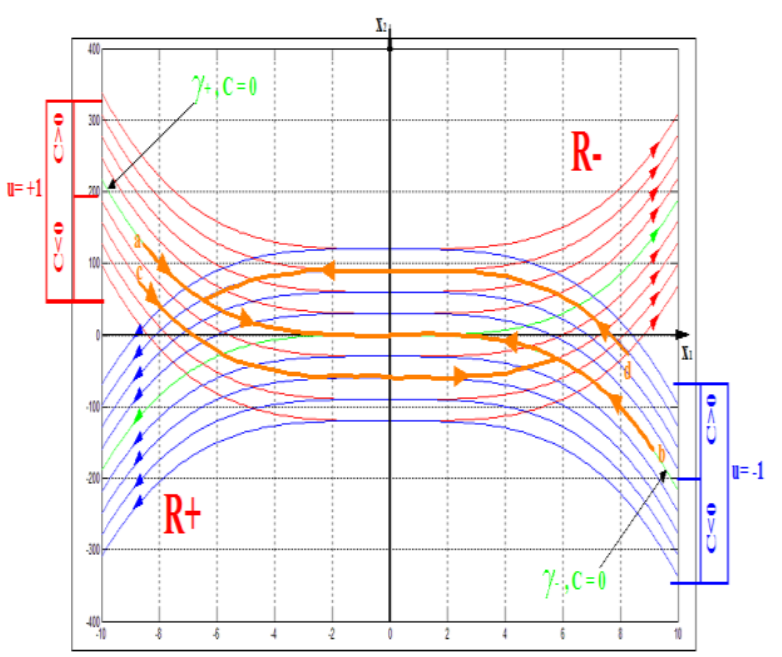

Figure 8: Various Trajectories by Four Possible Control Sequences

Figure 8: shows four possible control sequences (36) which drive the system from any initial condition to the origin.

1. If the system is initially anywhere (say a) on the $\gamma_{+}$

curve, the optimal control is $\mathrm{u}=+1$ to drive the

$$
t_{f}
$$

system to origin in minimum time

2. If the system is at rest anywhere (say b) on the $\gamma_{-}$

curve, the optimal control is $\mathrm{u}=-1$ to drive the

$$
t_{f}
$$

system to origin in minimum time

3. If the system is initially anywhere (say c) in the $R_{+}$

region, the optimal control sequence is $u=\{+$ $1,-1\}$ to drive the system to origin in minimum $t_{f}$

time

4. If the system is initially anywhere (say d) in the R.

region, the optimal control sequence is $u=\{-$ $1,+1\}$ to drive the system to origin in minimum $t_{f}$

time . If we start at $\mathrm{d}$ and use the control $\mathrm{u}=+1$ and use the optimal control sequence $u=\{-1,+1\}$, we certainly drive the system to origin but

$0.044]$ (a) we then have a control sequence $\{+1,-1,+1\}$ which is not a member of the optimal control sequence (34), and

$$
t_{f}
$$

(b) the time taken for the system using the control sequence $\{+1,-1,+1\}$ is higher than the $t_{f}$

corresponding time taken for the system with control sequence $\{-1,+1\}$.

- $\quad$ Step 9: Control Law: We now reintroduce * to indicate the optimal values. The time-optimal

$$
x_{1}, x_{2}
$$

control $\mathrm{u}^{*}$ as a function of the state [ , ] is given by

$$
\begin{aligned}
& \mathrm{u}^{*}=\mathrm{u}^{*}\left(x_{1}, x_{2}\right)=+1 \\
& \text { for all }\left(x_{1}, x_{2}\right) \in \gamma_{+} \cup R_{+} \\
& \mathrm{u}^{*}=\mathrm{u}^{*}\left(x_{1}, x_{2}\right)=-1 \\
& \text { for all }\left(x_{1}, x_{2}\right) \in \gamma_{-} \cup R_{-}
\end{aligned}
$$




$$
z=x_{2}-\left[0.064\left(x_{1}+\operatorname{sgn}\left(x_{1}\right) 0.201\right)^{2.5}-\operatorname{sgn}\left(x_{1}\right) 0.04 R\right. \text { ffferences }
$$

then if

$$
\begin{aligned}
& z>0, \quad u^{*}=-1, \\
& \text { And } \\
& z<0, \quad u^{*}=+1,
\end{aligned}
$$

- Step 10: Minimum Time: We can easily calculate the time taken for the system starting at any position in state space and ending at the origin. We use the set of equations (38) and (39) for each portion of the trajectory. It can be shown that the

$$
t_{f}^{*}
$$

minimum time for the system starting from ( , $x_{2}$

) and arriving at $(0,0)$.

\section{Conclusion}

The presented work was aimed to develop Optimal/Robust Control Strategy for Electrical Energy Cost Minimization in an Air-Conditioning Plant. We may summarize the work carried out as follows:

1. Modelling the thermal behaviour of buildings and designing an optimal control algorithm for their HVAC systems.

2. The system modelling order (seventh order system) has been simplified and approximated to another model of reduced order (second order system) that preserves the original transfer function as much as possible by using MATCHDC technique.

3. Dynamic optimal control algorithm that takes into account the time-varying behaviour of thermal loads and operates more efficiently and more economically.

4. The Phase Plane Trajectories for $\mathrm{u}=+1$ (red lines) and $\mathrm{u}=-1$ (blue lines) dedicated the direction of motion for increasing (positive) time.

5. The switching curve for Double Integral TimeOptimal Control System showed how to derive the

$$
\mathrm{x}_{1}(0) \mathrm{x}_{2}(0)
$$

system from any initial state ( , ) to final state at origin $(0,0)$ in minimum time.

6. Various trajectories control sequences showed that dynamic optimal control algorithm which is based on Pontryagin's Minimum Principle controller was capable of arriving different required states in minimum time.
1] B. Arguello-Serrano and M. Velez-Reyes. Nonlinear control of a heating, ventilating, and air conditioning system with thermal load estimation. IEEE Transactions on Control Systems Technology, 7(1):56-63, 1999.

[2] P. Norton and C. Christensen. A Cold-Climate Case Study for Affordable Zero Energy Homes. [Preprint].

[3] S.F. Larsen, C. Filipp'in, and G. Lesino. Thermal behavior of building walls in summer: Comparison of available analytical methods and experimental results for a case study. In Building Simulation, volume 2, pages 3-18. Springer, 2009.

[4] N. Mendes, G.H.C. Oliveira, and H.X. de Arau'jo. Building thermal performance analysis by using matlab/simulink. In Seventh International IBPSA Conference, Rio de Janeiro, Brazil, 2001.

[5] S. Bertagnolio and J. Lebrun. Simulation of a building and its HVAC system with an equation solver: application to benchmarking. In Building Simulation, volume 1, pages 234-250. Springer, 2008.

[6] Manohar R. Kulkarni, Feng Hong. Energy optimal control of a residential space-conditioning system based on sensible heat transfer modeling Building and Environment, 39 (2004) 31-38.

[7] Donald E. Kirk. Optimal Control Theory An Introduction. Dover Publications, Inc. Mineola, New York, 2004. 